\title{
Brilloun optical time domain analysis sensor assisted by a Brillouin distributed amplifier
}

\author{
Javier Urricelqui, Mikel Sagues and Alayn Loayssa \\ Universidad Pública de Navarra, Campus Arrosadia s/n, 31006 Pamplona, Spain.
}

\begin{abstract}
We demonstrate the extension of the measurement range of Brillouin optical time-domain analysis (BOTDA) sensors using a distributed Brillouin amplifier (DBA). The technique is based on injecting a DBA pump wave in the fiber to generate an additional Brillouin interaction that amplifies the BOTDA pump pulses. Furthermore, the differential pulse-width pair method is used to counteract the detrimental effects of the DBA amplification on the temporal shape of the pulses. Experimental proof-of-concept results in a 50-km fiber link demonstrate full compensation of the fiber's attenuation with no penalty on the signal-to-noise ratio of the detected probe wave.
\end{abstract}

Keywords: Brillouin distributed sensors, Brillouin optical time domain analysis, Brillouin amplification

\section{INTRODUCTION}

Brillouin optical time-domain analysis (BOTDA) sensors are a powerful tool for the distributed measurement of temperature and strain over large structures with very significant applications in civil and geotechnical engineering, in monitoring oil or gas pipelines or in assessment of high voltage cables. All these applications require a large sensing range. However, the sensing range of BOTDA sensors is essentially limited by the fiber's attenuation, which reduces the pump pulse power as it propagates along the fiber; thus reducing the Brillouin gain experienced by the probe wave and the signal-to-noise ratio (SNR) in detection. The obvious solution is to inject pulses with larger power in the fiber. However, this is ultimately limited by the onset of other non-linear effects such as modulation instability and Raman scattering. ${ }^{1}$ Another, more elaborate, solution is to amplify the pulses to compensate the attenuation of the fiber using distributed Raman amplification (DRA). ${ }^{2}$ However, DRA requires very high pump power (of the order of Watts) in the fiber, which can become an eyesafety concern in the installation and operation of real systems. Moreover, with DRA the relative intensity noise of the Raman pump laser is translated to the detected signal, significantly degrading measurement performance. ${ }^{2}$

In this work, we demonstrate the use of a distributed Brillouin amplifier (DBA) in BOTDA systems to amplify the pump pulses as they propagate along the fiber. This has advantages compared to DRA because a DBA requires much less pump power than DRA (milliwatts instead of watts) to achieve the same gain. Moreover, the amplification bandwidth can be precisely tailored to fit the pulse spectrum, instead of wasting the tens-ofnanometers bandwidth of DRA to amplify a narrow-band signal. Finally, the DBA noise added to the BOTDA signal is shown to be negligible.

\section{DESCRIPTION OF THE TECHNIQUE}

Fig. 1 schematically highlights the fundamentals of the technique depicting the various optical waves involved in the DBA-assisted BOTDA and their spectra. Three optical waves intervene: probe wave and pump pulses, used for sensing purposes as in any conventional BOTDA, and a additional wave (DBA pump), that is injected in the fiber and counter-propagated with the pump pulses. The DBA pump provides Brillouin gain to the pump pulses as they propagate along the fiber, thus compensating fiber attenuation. This requires the DBA pump wave to be offset from the pump pulse by an optical frequency difference that is around the mean Brillouin frequency shift (BFS) of the sensing fiber.

However, the Brillouin gain spectrum generated by a CW DBA pump would be very narrow, just the intrinsic Brillouin linewidth, which, for instance, would not be enough to amplify the pump pulses required for $1 \mathrm{~m}$ spatial

E-mail: alayn.loayssa@unavarra.es 


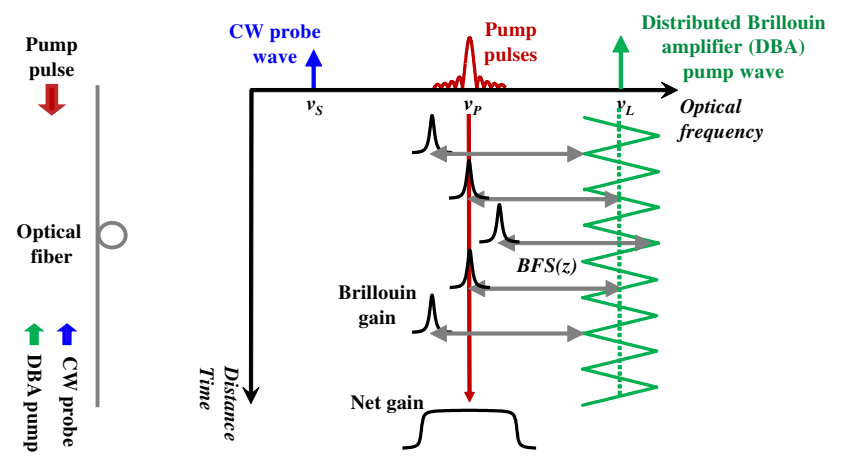

Figure 1: Spectra of optical waves present in the fiber for DBA-assisted BOTDA technique.

resolution. Therefore, there is a need to increase the DBA bandwidth beyond the natural Brillouin linewidth to properly amplify all optical spectral components of the pump pulse. This increment can be obtained modulating the wavelength of the DBA pump wave, as it is schematically depicted in Fig. 1. This makes the center wavelength of the Brillouin gain spectra experienced by the pump pulses to vary along the fiber. As a consequence, the pump pulse experiences a broad net gain spectrum. The wavelength modulation of the DBA pump is additionally synchronized to the injection of the pump pulses in the fiber, so that successive pump pulses experience the same gain at the same location; thus making the gain stable and avoiding the addition of noise to the BOTDA signal. Furthermore, the net gain can be made to have a flat frequency response with a judicious choice of wavelength modulation shape and frequency. ${ }^{3}$

\section{EXPERIMENTS}

Fig. 2 schematically depicts the setup used to demonstrate the technique, which is based on the conventional single-laser BOTDA scheme. A pulse generator drives a semiconductor optical amplifier (SOA) to generate optical pulses that are amplified and launched into the sensing fiber with a peak power of $21.79 \mathrm{dBm}$. In the probe branch, a Mach-Zehnder electro-optic modulator was used to generate two probe waves so as to compensate non-local effects. The power of each probe wave was $-6 \mathrm{dBm}$ and the deployed fiber was a $50-\mathrm{km}$ spool of standard single mode fiber.

The only additions to upgrade the conventional BOTDA for Brillouin gain assistance are the components that are highlighted in Fig. 2: an additional laser (DBA pump laser) and a signal generator synchronized to the pulses. The signal generator drives the current injection into the DBA pump laser to modulate its emission wavelength via laser's chirp. A triangular electrical signal with a frequency of $80 \mathrm{KHz}$ and amplitude adjusted to obtain a peak optical frequency deviation of $125 \mathrm{MHz}$ was used. The pump power for DBA injected in the fiber was $5 \mathrm{~mW}$. Finally, an optical filter before the detector is used to retain one of the probe sidebands and filters out the remaining DBA pump power.

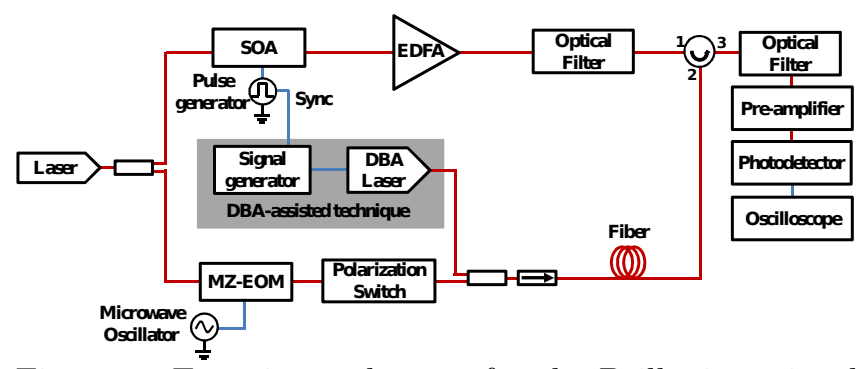

Figure 2: Experimental setup for the Brillouin-assisted BOTDA.

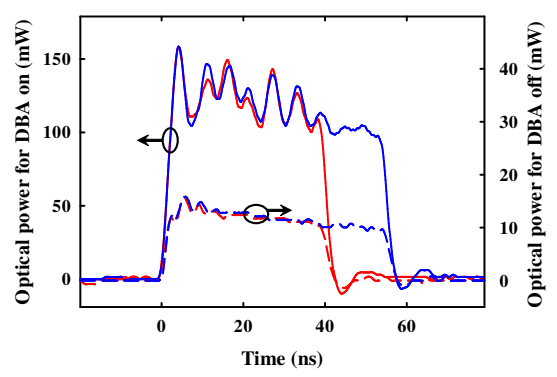

Figure 3: Amplification of pump pulses at the output of the fiber. 


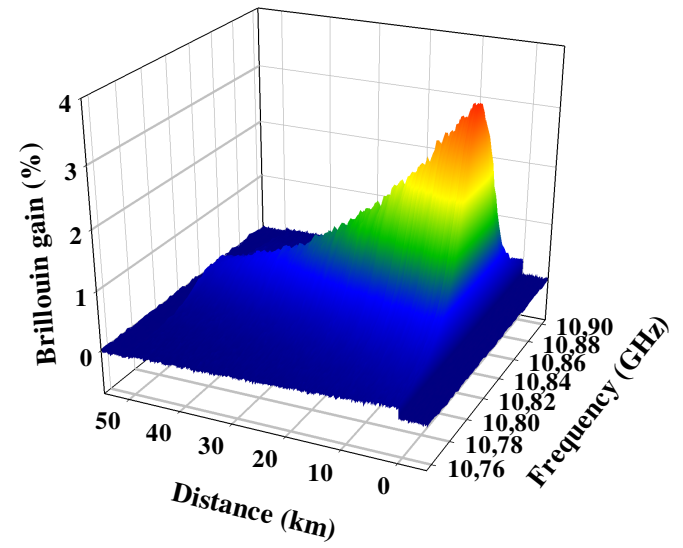

(a)

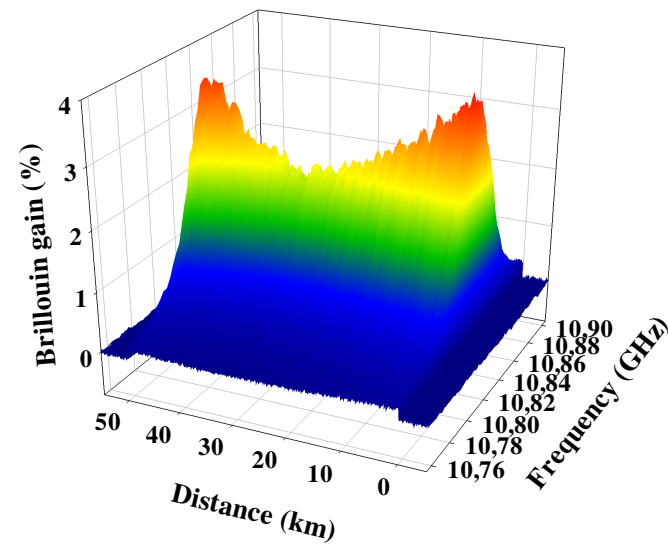

(b)

Figure 4: Measured distribution of the Brillouin spectra when the DBA is turned off (a) and on (b) for 55-ns pulses.

Fig. 4 compares the distribution of the Brillouin gain spectra measured along the fiber with the conventional BOTDA (DBA off) and the DBA-assited BOTDA. For conventional BOTDA, the amplitude of the Brillouin spectra decays exponentially due to the attenuation of the pump pulse as it propagates through the fiber. In contrast, when the DBA is on, the amplitude initially decays but starts to recover by mid-span as the DBA pump starts to kick-in. The net amplification for 40-ns and 55-ns pulses at the output of the fiber is shown in Fig. 3. Notice that in both cases a gain of around $10 \mathrm{~dB}$ is provided to the pulses, enough to compensated the $50-\mathrm{km}$ fiber attenuation.

Measurements of BFS for different duration pulses with the DBA turned on and off are displayed in Fig. 5. Note that, towards the end of the fiber, the BFS for the shorter pulses deviates from that measured with the conventional BOTDA. The shorter the pulse, the larger the deviation. This effect is attributed to the interplay between the temporal shape changes in the pulse brought by the distributed Brillouin amplification and the SPM effect. Stimulated Brillouin scattering is a dynamic phenomena that depends on the interaction of a pump and Stokes waves via an acoustic wave. The DBA pump is present at a certain location in the fiber and an acoustic wave is created and starts to grow exponentially when the pump pulse arrives, then it reaches an steady state (if the pulse is long enough) and, finally, the gain is abruptly cut-off as the pulse leaves. ${ }^{4}$ This alters the shape of the pulse as it is amplified along the fiber and makes it asymmetrical in time. Then, SPM comes into play generating an optical phase modulation that has been shown to increase the Brillouin gain linewidth when using symmetric pulses with large rise-time. ${ }^{5}$ In our case, with an asymmetrical pulse, SPM leads to down-shift of the instantaneous frequency of the pulse that translates into an apparent up-shift of the measured BFS. This effect is more pronounced for shorter duration pulses because the change in shape of the pulse takes place during the acoustic wave growth, which is of the order of the acoustic phonon lifetime. In Fig. 3 the pulse distorsion due to DBA is not very clear at first sight because it is masked to some extent by overshoot in the pulses caused by transient behaviour of the SOA, which also contributes to the asymmetry of the pulses and the detrimental effect of SPM. Moreover, further evidence on the role of SPM was confirmed by repeating the measurements with a reduced pulse power and observing that, in this case, the BFS profile matches to that of the conventional BOTDA.

We have devised a solution to compensate the BFS error due to SPM by deploying differential pulse-width pair (DPP) measurement, i.e. substracting the BOTDA traces for pulses of different duration, ${ }^{6}$ which are longer than the acoustic phonon lifetime. This takes advantage of the fact that temporal asymmetry in the pulses due to the transient behavior of the Brillouin amplification takes place at the start of the pulses during the acoustic wave growth. Furthermore, for pulses longer than the acoustic phonon lifetime, the pulse reaches quasi-steady state Brillouin amplification. Therefore, DPP measurement with two long-length pulses is not affected by SPMinduced shift in optical frequency and error in BFS. This was confirmed by experimental measurement of the BFS profile for DPP, which is superimposed to that of the conventional BOTDA in Fig. 5. 


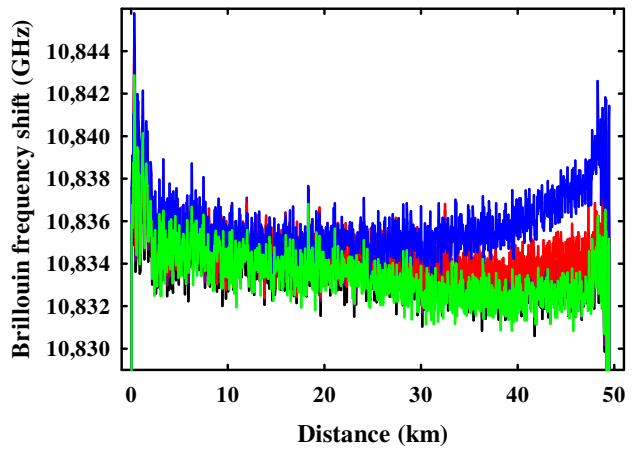

Figure 5: BFS distribution measured along the fiber with DBA for 15-ns (blue), 30-ns(red) and 40-ns (black) pulses and DPP of 40/55-ns(purple), and without DBA amplification for a $40-n s$ pulse (green).

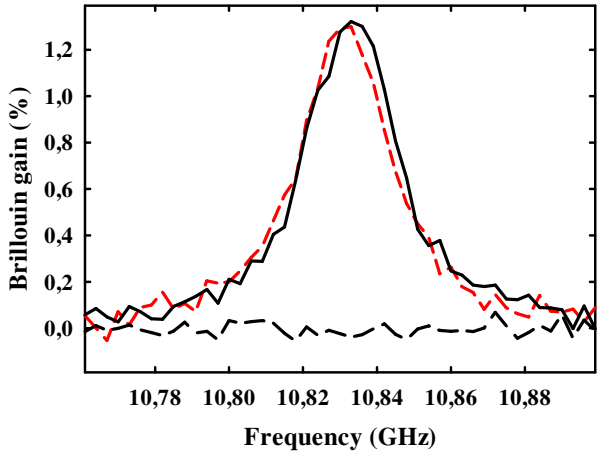

Figure 6: Brillouin gain spectra at the input (medium dashed red) and output (solid black) of the fiber link with DBA DPP using 40-ns and 55-ns pulses. Detection noise (long dashed black) without DBA.

Finally, Fig. 6 compares the measured DPP gain spectra at the input and output of the fiber. As it was expected, the amplitude of both spectra is similar (no normalization is used in the figure) as fiber attenuation has been compensated by DBA. Furthermore, the noise in both measurements was also found to have the same standard deviation as the noise detected without DBA. Just to visually compare them, the detection noise without DBA is also shown in Fig. 6. Therefore, it can be concluded that for these experiments the use of the DBA does not lead to any significant SNR penalty. This is a remarkable result specially compared to the use of Raman distributed amplification, which has been shown to add significant noise to the measurements. ${ }^{2}$ It can be explained by taking into account that Brillouin distributed amplification, despite having a large noise figure, just amplifies the pulses that counter-propagate with the DBA pump, but has no effect on the probe wave, particularly, no significant transfer of RIN noise. ${ }^{7}$

\section{CONCLUSION}

In summary, we have shown that the use of DBA amplification is a promising method to extend the range of BOTDA sensors having significant advantages in terms of required pump power, simplicity of implementation, and, more importantly, SNR.

We acknowledge support from the Spanish Ministerio de Economa y Competitividad project TEC2013-47264-C2-2-R, FEDER funds and the Universidad Pública de Navarra.

\section{REFERENCES}

[1] Foaleng, S. and Thévenaz, L., "Impact of Raman scattering and modulation instability on the performance of Brillouin sensors," Proc. of SPIE $\mathbf{7 7 5 3}$ (2011).

[2] Angulo-Vinuesa, X., Martín-López, S., Nuno, J., Corredera, P., Ania-Castanón, J., Thévenaz, L., and González-Herráez, M., "Raman-assisted Brillouin distributed temperature sensor over 100km featuring $2 \mathrm{~m}$ resolution and 1.2C uncertainty," J. Lightwave Technol. 30(8), 1060-1065 (2012).

[3] Zadok, A., Eyal, A., and Tur, M., "Gigahertz-wide optically reconfigurable filters using stimulated Brillouin scattering," J. Lightwave Technol. 25(8), 2168-2174 (2007).

[4] Beugnot, J.-C., Tur, M., Mafang, S. F., and Thévenaz, L., "Distributed Brillouin sensing with sub-meter spatial resolution: modeling and processing," Opt. Express 19(8), 7381-7397 (2011).

[5] Foaleng, S., Rodríguez-Barrios, F., Martín-López, S., González-Herráez, M., and Thévenaz, L., "Detrimental effect of self-phase modulation on the performance of brillouin distributed fiber sensors," Opt. Lett. 36(2), 97-99 (2011).

[6] Li, W., Bao, X., Li, Y., and Chen, L., "Differential pulse-width pair BOTDA for high spatial resolution sensing," Opt. Express 16(26), 21616-21625 (2008).

[7] Zhou, J., Chen, J., Jaouen, Y., Yi, L., Petit, H., and Gallion, P., "A new frequency model for Pump-to-Signal RIN Transfer in Brillouin Fiber Amplifiers," IEEE Photon. Technol. Lett. 21(13), 978-980 (2007). 
Copyright 2015 Society of Photo Optical Instrumentation Engineers. One print or electronic copy may be made for personal use only. Systematic reproduction and distribution, duplication of any material in this paper for a fee or for commercial purposes, or modification of the content of the paper are prohibited. 